\title{
Application Design of Quality Management of Assembly Workshop Process
}

\author{
Wang Yan, Zhang Jianmin, Zheng Chao \\ Information and Software Development Center, Beijing Institute of Aerospace Control Devices, Beijing, China
}

Email address:

657961532@qq.com (Wang Yan)

\section{To cite this article:}

Wang Yan, Zhang Jianmin, Zheng Chao. Application Design of Quality Management of Assembly Workshop Process. Science Discovery. Vol. 9, No. 2, 2021, pp. 85-90. doi: 10.11648/j.sd.20210902.21

Received: March 17, 2021; Accepted: April 22, 2021; Published: April 26, 2021

\begin{abstract}
China's manufacturing industry is developing rapidly. Quality management has become an important link in industrial production and operation activities. The level of workshop quality management has a profound impact on the improvement of production efficiency. The use of information technology to design the quality management application system of the assembly workshop process and solve the problems exposed in the quality management process is of great significance to improve the assembly efficiency of the workshop. In the previous workshop assembly process, offline information transmission was inefficient and error-prone. In response to this risk, this article develops a process quality management system for the assembly workshop, which enables the assembly work and quality management work in the workshop to work together. so that the information can be quickly transmitted and responded to. Therefore, this system guarantees high-quality and high-efficiency production of products. At the same time, a quality management model framework was built. And functions such as production line management, schedule efficiency analysis, quality inspection traceability were completed. It has realized the organic integration of efficient and coordinated operations of operators, quality management, planning and materials in the workshop assembly process. Based on this, the design of the quality management information system for the assembly workshop was completed, which provided strong support for the digital integration of the system, big data processing and intelligent manufacturing.
\end{abstract}

Keywords: Workshop Assembly, Process Quality Management, Efficient and Collaborative Operation

\section{装配车间过程质量管理应用设计}

王岩, 张健敏, 郑超

北京航天控制仪器研究所信息化与软件开发中心, 北京, 中国

邮箱

657961532@qq.com(王岩)

摘要: 我国制造业发展迅速, 质量管理已成为了工业生产经营活动中的重要环节, 车间质量管理水平对生产效率有巨 大的影响。利用信息化手段设计适用于车间的质量管理应用系统，解决质量管理过程中暴露的问题，对提升车间装配 效率具有重要意义。以往的车间装配过程中，线下信息传递效率低且易出错。针对此风险，本文开发一套装配车间过 程质量管理系统，使车间的装配工作和质量管理工作能协同运作，使信息能迅速传递响应，进而保障产品高质量、高 效率生产; 同时构建了质量管理模式框架, 完成了生产线管理、进度效率分析、质量检验追溯等功能, 实现了车间装 配过程中操作工、质量管理、计划与物料等高效协同运作的有机整合。基于此，完成装配车间质量管理信息系统的设 计, 为系统数字化集成、大数据处理及智能制造提供了强有力的支撑。 
关键词: 装配车间, 过程质量管理, 高效协同运作

\section{1. 引言}

目前, 我国的车间生产信息化管理系统建设普遍集中 在企业三层集成模型中。它能够通过有效的互相响应, 控 制车间生产线设备的状态，制定更加高效的生产方式和工 艺流程, 实现在车间生产过程中对零组件状态的实时监控、 对产品状态的报工检验与质量追踪, 为后期的计划排产和 生产方式改进等提供真实、有效的依据，从而进一步使装 配车间的生产过程形成一个完整的信息流[1-2]。企业三层 集成模型如图1所示:

\begin{tabular}{|c|c|}
\hline 企业资源管理（ERP） & 计划层 (企业级) \\
\hline 制造执行系统（MES） & 执行层 (生产级) \\
\hline 过程控制层 (Control) & 控制层 (过程级) \\
\hline
\end{tabular}

图1 企业三层集成模型。

其中, 在 $\mathrm{B} / \mathrm{S}$ 架构基础上开发的基于ASP.NET质量管 理系统实现了检验实验室质量管理体系实时化、信息化, 也满足了对体系远程监管的需求。该系统具有样品管理、 质量控制、体系审批、设备管理、药品管理和后勤管理等 模块, 并建立了标准关系数据库[3]。

军工企业相较于其他企业而言存在一定的特殊性, 潜 移默化地影响着国家国防事业前进与发展, 在新形势背景 下, 军工企业升级了军工产品质量管理系统, 对影响企业 实施质量管理体系认证有效性的因素进行了分析, 并进一 步指出了保证企业实施质量管理体系有效性的四个关键 因素[4]。

相关领域的车间质量管理系统通过对质量数据的实 时采集、统计分析及处理, 针对智能化生产过程中对产 品质量的可控性需求, 提出一种基于MES的产品质量管 理系统, 解决质量信息流通不畅、反馈滞后等问题, 实 现对产品质量和过程质量的实时监控, 并能在工序质量 出现异常时启动报警, 同时给出相应的处理意见[5]。基 于企业三层集成模型的车间的信息化管理系统建设在领 域内蓬勃发展, 面向质量管理的多行业研究也在大力发 展。

当装配车间的规模发展到一定程度时，高效质量问 题解决方案就变成了让生产效率和质量提升的基础, 同 时解决质量问题的路也变得越来越困难。因此, 车间要 求质量管理体系的运行要更加合理高效。想要具备快速 响应、高效运转的全流程周期工序工步质量问题分析能 力, 改进现有的质量问题处理方案, 实现装配车间过程 质量管理水平的提高, 就要实现装配车间质量管理的信 息化建设。

现有的车间装配流程缺少与质量管理工作之间的 数据交互关系, 将信息化的质量管理系统应用于装配车 间生产过程，可以提高车间管理的水平，形成高效协同
运作的数据信息交互关系, 进而提升生产效率, 降低成 本。

本文提出了质量管理系统在装配车间过程上的应用 设计, 保证了质量管理的透明化、高效化, 确保了数据信 息的实时同步和准确, 提高了车间装配生产线的生产效率, 降低了不良产品的流出率, 便于统计零组件在整个生产周 期的状态, 从而促进了生产装配车间的数字化转型, 实现 了装配车间与应用系统的整体优化。

\section{2. 装配车间过程的质量管理系统}

本文结合质量管理系统在国内外研究综述的基础上, 设计与开发装配车间自身的质量管理系统, 将各部门间的 质量管理工作有机合理的结合起来, 提高装配车间生产效 率, 降低物料的消耗, 保持生产零组件产品质量的稳定, 提高零组件产品的质量水平。

\section{1. 质量管理系统总体研究过程}

本课题重点研究装配车间过程质量管理系统的开发 与应用, 主要研究过程如下:

\subsection{1. 质量架构分析}

分析当前装配车间所使用的信息传递系统的功能架 构, 结合质量管理体系要求, 探索装配车间的自主质量 管理模式。研究质量管理系统与装配车间的信息交互方 式, 设计质量管理系统的总体功能。在完善现有的质量 管理体系与信息传递方案的基础上, 保证新的质量管理 体系能够有效地运行, 建立标准化、规范化的质量管理 系统。

\subsection{2. 建立质量跟踪卡填报技术}

通过结合装配产品质量跟踪卡里面每个工序下的工 步快速报工数据、检验结果与检查项、零件、原材料、外 协外购、设备等数据的填报次序与填报内容, 分析生产工 序的状态的同时设计质量跟踪卡填报方案, 通过进度的追 踪, 为质量控制提供参考, 将可能出现的问题隐患解决在 质量跟踪卡填报前段, 避免后期损失。

\subsection{3. 设计装配车间过程质量管理系统}

基于装配车间的质量管理系统需求分析, 对各功能结 构进行设计, 根据车间的装配业务流程, 逐步分解流程, 建立工作流模型, 实现车间质量管理的质量控制与管理、 质量数据的科学分析、质量预警与质量追溯等功能。实现 车间装配任务与质量管理系统的信息交互和高效协同运 作。

\subsection{4. 开发系统}

系统实现了装配车间的过程质量管理的应用设计, 应 用Mysq1-5.7.21数据库服务器进行了车间装配生产现场的 
数据表、字段信息的存储, 利用Java和JavaScript实现系统 过程质量管理的网页系统页面与功能模块, 对质量跟踪卡 质量管理、装配进度及车间装配状态的质量控制等进行及 时、准确、全面的监控和管理。

\section{2. 质量管理系统重点模块框架}

质量管理模块的主要包括: 工艺管理、基础数据管 理、工序工步质量控制及其子内容的填报、装配流程质 量管理和质量统计分析。还包括工序工步的报工、质量 检验管理、质量跟踪卡完成进度可视化展示、统计分析 内容导出等内容。基于质量控制与检验的质量管理系统 如图2所示:

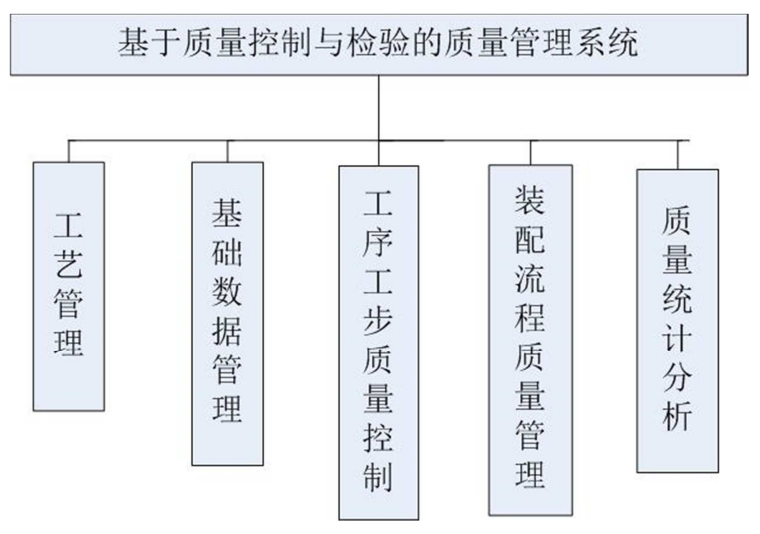

图2 基于填报与检验的质量管理模块。

结合装配车间的业务需求, 本过程质量管理系统满足 如下功能:

对质量跟踪卡、员工用户个人信息、物料设备、检验 标准、数据表等基础信息的统一管理, 为标准化的管理提 供基本保障。

质量管理业务流程规范化、标准化，质量业务的分级 审理和分类处理，相关联质量问题的整合，通过整合装配 车间质量问题数据, 便于提出切实可行的解决方案。

借助车间装配过程中的数据, 对零组件的批次、物料 信息、供应商信息作为条件查询, 将相关信息进行产品全 生命周期质量追溯。

将质量跟踪卡等生产过程信息形成质量报表，对整体 质量进行合理控制, 形成便于质量信息快速检索的质量文 档[6]。

系统中装配车间操作流程的核心是质量控制和质量 管理, 质量跟踪卡中包含着零组件制造所需要的工序、工 步、检查项等步骤的填报与检验, 操作工完成相应的车间 装配任务后, 在质量管理系统上进行填报。填报的实时数 据通过系统进行实时采集, 并将实时采集的填报数据与报 工数据保存到数据库中。质量控制和质量管理的功能模块 图如图3所示:

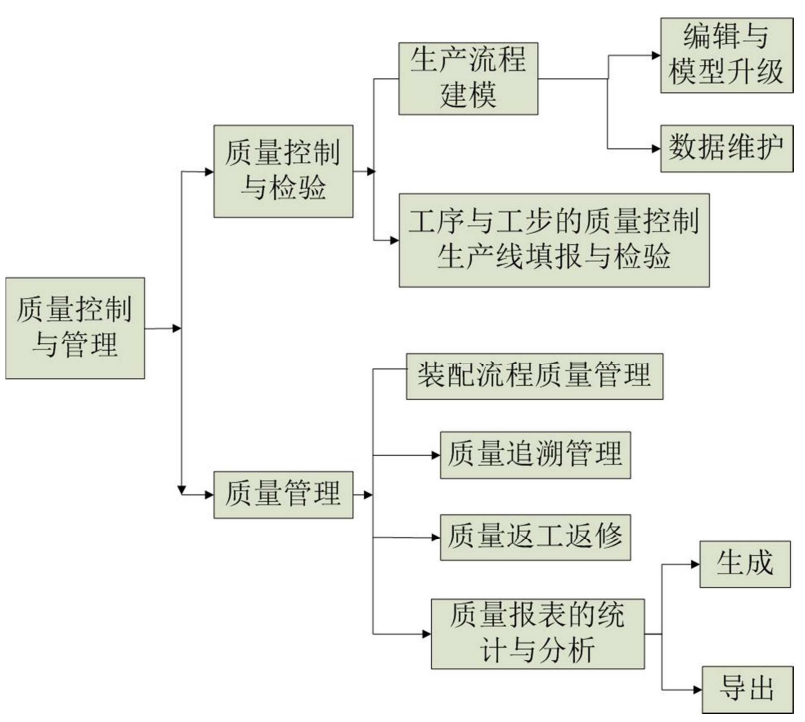

图3 质量跟踪卡的功能模块图。

\section{3. 质量跟踪卡实例的进度可视化显示模块}

质量跟踪卡的实例管理模块用于质量控制、报工和填 报, 可以通过计划名称查询具体质量跟踪卡实例内容。通 过名称或质量编号查询, 可以显示具体工步报工进度。

在质量控制与检验管理模块中，根据生产线填报的内 容, 可进入质量控制的实例信息列表中进行信息查看, 实 现质量控制中填报内容的判读、工步的报工和质量跟踪卡 的统计报表生成和导出等操作。

为了实现更加紧凑的信息传达，把操作工填报的信息 与检验员获取的信息有效结合, 解决车间装配节奏慢的问 题。监控整个车间装配线的质量控制进度信息, 快速定位 装配车间生产线的步骤次序问题[7]。

质量跟踪卡的报工、检验进度可视化功能, 在操作工 与检验员工作区域的计算机上部署系统显示。进度可视化 的界面部署在质量控制信息列表中，同时将生产线中工序 与工步的填报校验功能和装配流程的质量管理相结合 [8]。 在此界面中, 包含产品编号和报工进度的质量跟踪卡实例 进度可视化显示模块如图4所示:

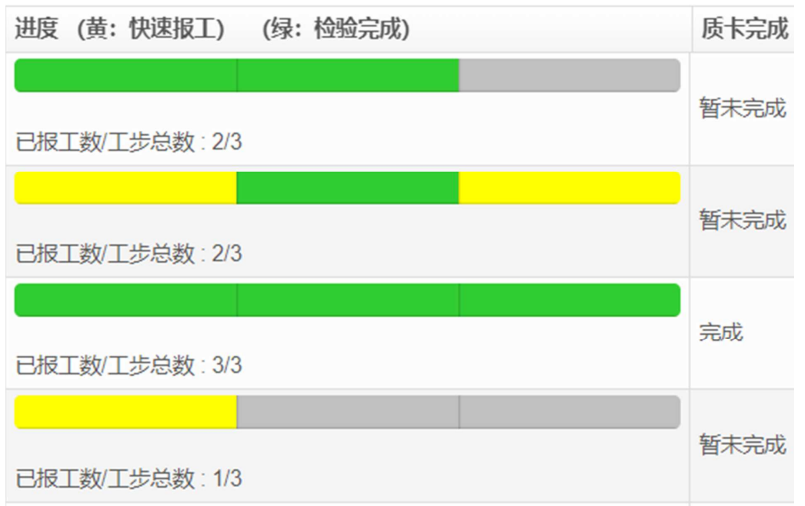

图4 质量跟踪卡实例进度可视化显示模块。

当车间装配报错、产生质量问题, 或需要统计错误出 现频率较高的工步时, 进度可视化模块的显示屏上对应的 
工步将有明显的颜色区分, 便于快速统计问题, 组织会议, 进行问题解决方案研讨。

当相应的检查项等信息填报完成且工步报工完成后， 检验员的可视化界面也能够清楚看到待检验信息, 节省了 点击质量跟踪卡填报详情逐一查看的装配时间。

通过此逐层自底向上的报工、检验、进度可视化的高 校协同工作, 装配车间的管理者可以简单便捷的了解到车 间实时生产进度以及未完成工步的状态, 并及时解决问题。 同时, 管理者可以通过对实时装配车间生产线的状态和质 量跟踪卡完成进度的分析, 对关键步骤进行改良与处理, 提升工作效率[9]。

\section{4. 质量跟踪卡的生成及导出}

质量跟踪卡实例操作中最主要的功能是质量跟踪卡 导出和合并, 形成质量报表。该实例支持生成、导出、合 并等功能, 并在汶览器弹出文件。

单条质量跟踪卡的质量报表，直接在预设路径中生 成.doc格式的质量跟踪卡文件, 同时导出下载到汶览器本 地。质量跟踪卡实例同时支持批量生成、导出与合并, 多 条质量跟踪卡的质量报表, 导出汶览器弹出.zip文件压缩 包, 包含着按照质量报表的编号排序的多个.doc格式的文 件。导出的单条质量跟踪卡文件内容格式如表1所示。

表1 车壳涂层漆料装配质量跟踪卡。

\begin{tabular}{llllll}
\hline 工序 & 工步 & 技术要求 & 检查结果符合要求 & 操作者 \\
\hline 工序1 & 工步1 & 自检 & 互检 \\
工序1 & 工步1 & 检查项1 & 是 & 系统管理员 & 互检员 \\
工序1 & 工步1 & 检查项2 & 1.0 & 系统管理员 & 互检员 \\
工序1 & 工步1 & 检查项3 & 是 & 系统管理员 \\
工序1 & 工步1 & 检查项4 & 5 & 自 & 检员 \\
工序1 & 工步1 & 检查项5 & 理员 & 互检员 \\
\hline
\end{tabular}

\section{5. 检查项填报的校验}

检查项填报具有防错校验功能, 任一步骤的检查项出 现错误填报范围均不会提交成功进行下一步操作, 只有填 入正确范围内的值, 才能将数据记录到数据库中。

整个质量跟踪卡填报过程完成之后, 选择生成质量跟 踪卡并导出功能, 系统会打印出相应的质量跟踪卡, 打印 成纸质版进行保存, 与质量管理结合作为质量报表的一部 分。这种方式与线下人工填写记录相比较, 准确度更高、 效率提升巨大。在车间装配与生产线填报过程中的各种质 量数据、设备状态、检验结果信息, 会通过系统传输并保 存到系统后台, 便于质量管理者查看数据, 进行数据分析, 同时也便于后期生产与质量追溯管理和质量返工返修。

\section{3. 过程质量管理的应用场景}

\section{1. 车间装配实时参数监控}

由于重要的关键工序参数、强检点参数和附表等关键 的参数信息内容在完成生产线填报后, 测量结果和检验结 果可以实时反映到现场系统的质量跟踪卡实例进度可视 化显示模块上, 因此, 操作工与检验员等工作人员可以及 时发现异常的填报内容、错误点和潜在的失效可能, 更早 的进行质量问题预防, 进行装配线调整等反应, 避免装配 完成的产品出现质量问题，从而导致大量返工[10]。

除此之外，当出现填报错误或无法顺利导出质量报表 等特情况导致工程师无法即刻到装配车间现场工作时, 车 间的实时监控参数列表也能为其远程调试、锁定现场问题 提供了可行性[11]。

车间装配实时参数监控在系统中还有另外一个模块 应用, 即“本周在线人数”模块, 该模块可以显示自当日起 七天内的系统登陆人次情况统计折线图, 如图5所示。随 着不同身份和权限用户的每次登录, 在该模块中实时更新
并显示当日累计在线用户数量、本周登陆人次统计图等统 计功能, 便于车间进行装配的同时, 进行实时登陆人员、 操作人员的监控与调整。此模块作为实施参数监控的一部 分, 可将折线图保存为图片, 导出并归入质量报表统计文 档中。

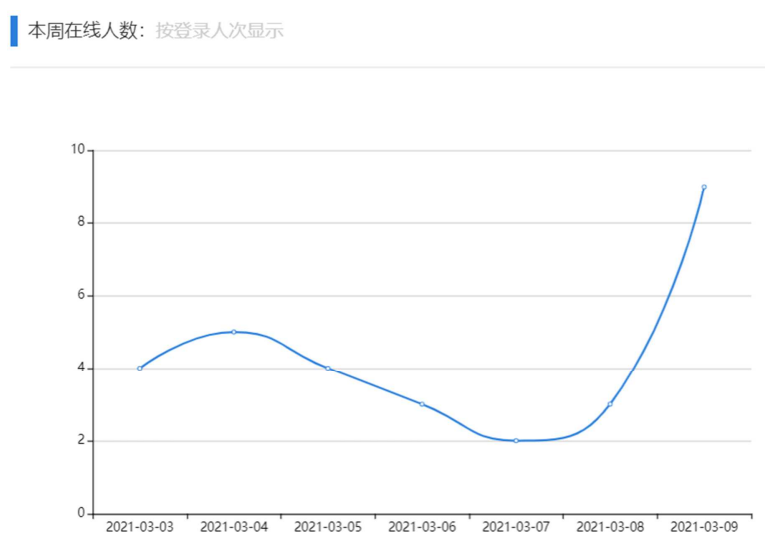

图5 本周在线人数模块。

\section{2. 班组会议和日常管理会议}

每个阶段性车间装配任务结束和开始的时候, 通过本 装配车间质量管理应用系统, 将重要的零组件装配生产信 息、生产指标的完成情况和需要解决的问题点, 及时展示 给相应负责的班组成员, 并在日常的部门、班组例会和质 量管理会议上沟通讨论并寻求解决方案。

在日常管理会议上, 通过对装配车间管理层制定的装 配指标进行回顾, 确认下一阶段需要重点关注的工作内容, 并采取工作措施。通过对投产计划、历史投产比例和完成 情况比例等情况的结合, 及时组织会议, 完成对装配过程 中遇到的偏差的纠正和对问题的闭环研究 [12]。

车间质量管理日常例会如表2所示。 
表2 车间质量管理日常例会。

\begin{tabular}{llll}
\hline \multirow{2}{*}{ 负责人 } & \multicolumn{3}{l}{ 车间质量管理例会 } \\
\cline { 2 - 4 } & 会议名称 & 会议内容 & 输出及跟踪 \\
\hline 质量评审员 & 质量评审 & 质量问题评审确认 & 车间落实 \\
操作工 & 生产例会 & 车间装配问题沟通 & 成产报表 \\
生产负责人 & 指标回顾 & 上月完成情况分析 & 回顾记录 \\
工程师 & 复杂问题解决 & 问题汇报与解决 & 质量报表文档 \\
\hline
\end{tabular}

\section{3. 过往装配情况的追查}

当装配完成的零组件出现生产质量问题时, 可以通 过质量管理系统追溯装配过程的任何一个环节，根据填 报与检验的情况确定问题所在的工步。通过分析, 若是 零件、原材料、外协外购件、设备零件等生产制造过程 的问题, 可以通过追溯来反查相应的零部件批次信息; 若是装配的问题, 可以从质量跟踪卡详情入口进入，反 查相应零组件的过程参数和检测结果信息。通过质量跟 踪卡的详细信息显示, 为进一步了解装配质量问题来源 提供极大的可靠性，带来了便利的同时，节约了时间成 本和人力投入。

综上所述, 装配车间过程质量管理系统的设计与应用 作为强有力的技术支撑，对于强化装配车间的信息化管理 有着重要意义, 能够切实提升车间的生产效率, 降低工作 人员的时间成本 $[13]$ 。

\section{4. 过程质量管理系统应用的成果}

\section{1. 质量管理应用对计划管理的提升}

装配车间过程质量系统的质量管理体系运行后, 在装 配车间的电子化管理工作中具有重要意义。车间企业的核 心内容便是生产, 因此在生产基础上, 强化生产计划管理、 子计划下发、个人任务管理，做好车间装配计划下发的方 案也是必要的。随着质量管理系统的部署, 与质量问题紧 密关联的计划模块也产生了效率提升。

计划管理的功能模块图如图6所示:

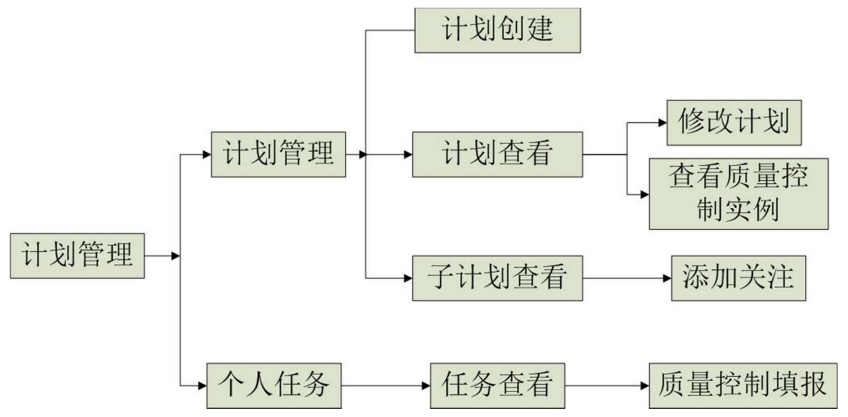

图6 计划管理的功能模块图。

在编制相应的车间装配计划时，应综合考虑车间企业 的月度计划和全年计划，将装配、填报、检验任务和质量 控制与管理任务按日分解，并在装配计划的基础上，做好 全部准备工作，为后续装配车间的顺利生产奠定坚实的基 础。
操作工、调度员、检验员等相关工作管理人员, 可以 依靠装配车间质量管理系统, 对生产任务进行及时了解, 并结合系统中质量管理列表页面所反馈的装配实际情况, 安排好装配计划。使得车间管理人员能够在系统所提供的 信息的基础上确定好生产装配流程以及生产计划中所规 定的生产精度、合格率以及材料使用情况, 明确产品、零 组件装配完毕的具体时间、质量跟踪卡填报时间, 合理安 排计划[14]。

负责计划下发的工作人员凭借物料模块提供的信息， 与物料库管员沟通了解车间物料库存情况, 基于此深入分 析车间实际的装配能力, 再根据个人能力库的内容可以初 步通过系统计算并判断车间是否能够按时完成当月的生 产装配任务。与此同时, 工作人员还能根据车间实际反馈 的情况, 科学调整质量控制与管理内容, 切实提升车间装 配计划的可操作性和可行性。

\section{2. 过程质量管理系统部署的效益分析}

部署装配车间过程质量管理系统解决方案后, 效益分 析如下:

车间装配流程自动化。通过使装配任务与生产计划高 效协同运作，优化了车间装配的生产过程并提升产能，提 升了车间各工序间生产连续性、协同性, 减少车间装配过 程中的低效等待时间, 减少额外的数据确认时间, 进而使 车间装配流程自动化程度提高[15]。

人力投入的降低。质量管理系统部署前, 产出、报废 数量、产品过程参数、测量值等生产和质量数据需要由现 场操作工填写在纸质文档上, 再额外增设一名数据员将纸 质文档上的数据录入部门电子文档。在部署系统后, 由于 生产数据直接由系统抓取, 可以节约工作人员的大量工作 时间, 同时避免了人为的填写错误, 提升质量报表的准确 性并提高现场无纸化率，降低人力投入。

经济效益的提升: 消除车间操作工与检验员的非增值 工作, 提高工作效率和劳动生产率, 通过质量管理数据的 即时透明显示, 极大降低了出现质量事故的风险, 提升经 济效益[16]。

\section{5. 结论}

本文提出了装配车间过程质量管理的应用系统，设 计开发完成并投入运营使用，解决了车间装配过程中装 配进度上报速度慢、装配进度无法及时同步导致的无效 生产时间过长、生产效率较低等问题。应用表明，装配 车间过程质量管理系统能够通过质量控制与管理、生产 线填报与检验、进度可视化显示模块和车间装配实时参 数监控等功能, 将车间装配现场的实时进度信息准确的 转递给其他操作工和质量检验员, 使装配信息高效运转, 可极大提高车间质量管理水平，显著提升生产效率和产 品质量。

本设计方法经调试成功，投入车间装配系统的装配质 量管理生产线使用后，系统运行稳定，各部门高效协同运 作, 满足了车间的信息化要求, 使过程质量管理透明化、 高效化，提升了车间装配生产线的生产效率。 


\section{参考文献}

[1] 吴卯恩,胡晓轩,李革. 光伏产业标准化智能工厂透明化的 实践与前景 $[\mathrm{A}]$. 中国标准化协会.第十七届中国标准化论 坛论文集[C].中国标准化协会:中国标准化协会,2020:9.

[2] 乔运华,何山,王鹏, 李文博,刘晨燕.车间制造执行系统 (MES) 在电子装配行业大批量批次生产模式下的应用研究——以 $\mathrm{J}$ 公司为例 $[\mathrm{J}]$.制造业自动化,2020,42(11):71-74.

[3] 李正华. 基于ASP.NET的检验实验室动态质量管理系统 [J]. 电脑知识与技术,2019,15(19):87-89.

[4] 夏晓雷.军工产品的质量管理系统优化探讨 [J]. 科技 风,2019(01):94.

[5] 张蕾.基于MES的产品质量管理系统的设计与研究 [J].轻工 科技,2019,35(10):102-103.

[6] 周子涵,高瑜雄.系统在制造生产企业中的应用与研究 [J].软 件,2020,41(11):214-216.

[7] 游健平.生产车间生产管理信息系统设计与构想 $[\mathrm{J}]$. 电脑知 识与技术, $2020,16(30): 78-79+84$.
[8] 任耀华.面向光伏电池数字化车间的系统设计 [J]. 电子工业 专用设备,2020,49(04):50-53.

[9]袁明锋,杨否,李通,祥保玉.基于系统的质量信息提取及应用 [J].新技术新工艺,2020(07):78-80.

[10] 费一正,汪惠芬,刘婷婷,孔正.基于 $\mathrm{SPC}$ 的车间制造质量管理 系统研究[J].制造业自动化,2011,33(19):43-46.

[11] 李崇.制造执行系统在数控车间中的应用 $[\mathrm{J}]$.信息技术与标 准化,2020(11):61-66.

[12] 董炳,梁荣,管兵,薛江段,商英.基于IATF 16949对涂装车间 质量管理的优化 [J].现代涂料与涂装,2020,23(03):37-39.

[13] 马丽丽.电子装配行业的车间制造执行系统 (MES) 应用 [J]. 制造业自动化,2019,41(12):48-51.

[14] 陈海东,左玮,孙志华.全流程质量管理信息化系统应用研究 [J].经济研究导刊,2020(02):182-183.

[15] 李烈强. 印制线路板表面贴装生产线质量管理研究[D]. 重 庆大学,2019.

[16] 申伟.质量管理系统在特种设备检验检测中的应用探讨 [J]. 化学工程与装备,2019(12):218-219. 